hep-th/0609083

\title{
Amplitudes With Different Helicity Configurations Of Noncommutative QED
}

\author{
Abolfazl Jafari \\ Institute for Advanced Studies in Basic Sciences (IASBS), \\ P. O. Box 45195, Zanjan 1159, Iran \\ jabolfazl@iasbs.ac.ir
}

\begin{abstract}
The amplitudes of purely photonic and photon-2-fermion processes of noncommutative QED (NCQED) are derived for different helicity configurations of photons. The basic ingredient is the NCQED counterpart of Yang-Mills recursion relations by means of Berends and Giele. The explicit solutions of recursion relations for NCQED photonic processes with special helicity configurations are presented.
\end{abstract}

Keywords: Noncommutative Geometry, Noncommutative Field Theory

PACS No.: 02.40.Gh, 11.10.Nx, 12.20.-m 


\section{Introduction}

The calculation of cross sections for the production of many particles in high-energy collisions is restricted by the technical difficulties associated with the evaluation of the corresponding multi-particle Feynman diagrams. In contrast to the usual approach, one can express the amplitudes in terms of positive and negative chirality spinors $(\lambda$ and $\tilde{\lambda})$ and their corresponding products. It is known that the amplitudes associated to special helicity configurations have very simple expressions in terms of these spinor products [1, 2, 3, 4, 5].

Much significant progresses have been made in the last years with the development of new techniques, principally the Maximally Helicity Violating (MHV) rules introduced by Cachazo, Svrcek and Witten (CSW) [6], and also the the recursion relation among amplitudes by Britto-Cachazo-Feng (BCF) [7]. CSW introduced a novel diagrammatic technique, MHV rules, in which maximally helicity violating amplitudes are used as vertices in a scalar perturbation theory, for which the ver-

tices are connected by scalar propagators $\frac{1}{p^{2}}$. This arrangement vastly reduces the number of diagrams that must be evaluated relative to the traditional Feynman rules case. Although the original CSW paper dealt only with purely gluonic amplitudes, the formalism has been successfully extended to include quarks Higgs and massive gauge bosons.

The focus of attention has been on developing and exploiting techniques for QCD scattering processes, since such amplitudes are needed to estimate multi-jet cross sections at hadron colliders [4, 8, 9, 10, 11]. Indeed, the MHV rules were specifically developed for and applicable to massless Yang-Mills field theory. As mentioned, particular helicity amplitudes in Yang-Mills theory take on unexpectedly simple forms [12, 13, 14, 15]. At tree level for example, purely gluonic colure-ordered scattering amplitudes can be summarized as follows

$$
\begin{aligned}
& \varepsilon(1 \pm, 2+, \cdots, n+)=0 \\
& \varepsilon(1-, 2-, \cdots, n+)=\frac{\langle 12\rangle^{4}}{\prod_{k=1}^{n}\langle k k+1\rangle}
\end{aligned}
$$

So amplitudes with all the gluons having the same helicity vanish, as do those with only one gluon having a different helicity to the others. The second case above therefore corresponds to the maximally helicity violating amplitudes. Their simple forms were first conjectured by Parke and Taylor [16], and later proven by Berends 
and Giele using a recursive technique [2]. The amplitude

$$
\varepsilon(1+, 2+, \cdots, i-, \cdots, j-, \cdots, n+)
$$

is called a "mostly plus" MHV amplitude, for obvious reasons. Its mostly minus counterpart, which has two positive helicities and the remainder negative, is called an $\overline{\mathrm{MHV}}$ amplitude, and can be obtained by interchanging $\langle i j\rangle \rightarrow[i j]$. Another example is

$$
\begin{aligned}
\varepsilon(\bar{f}+, f-, 1+, 2+, \cdots, n+) & =0 \\
\varepsilon(\bar{f}+, f-, 1+, 2+, \cdots, i-, \cdots, n+) & =\frac{2^{\frac{n}{2}} e^{n}\langle f \bar{f}\rangle^{n-2}\langle f i\rangle^{3}\langle\bar{f} i\rangle}{\prod_{k=1}^{n}\langle f k\rangle\langle\bar{f} k\rangle}
\end{aligned}
$$

Here $i^{+}$denotes a positive helicity photon with momentum $p_{i}$ and $f, \bar{f}$ denote fermion and anti-fermion respectively. This is the fundamental MHV amplitude in QED, and as before it consists of only a single term. The factor $e^{n}$ is the gauge coupling constant 4 .

On the other hand, recently a great interest has been appeared to study field theories on spaces whose coordinates do not commute. These spaces, as well as the field theories defined on them, are known under the names of noncommutative spaces and theories. In contrast to $\mathrm{U}(1)$ gauge theory on ordinary space-time, as we briefly review in next section, noncommutative version of theory is involved by direct interactions between photons. Interestingly one finds the situation very reminiscent to that of non-Abelian gauge theories, and then the question is whether the techniques developed for non-Abelian theory purposes can be used for noncommutative QED case too. In particular, the same question may arise for the recursive relation techniques [17, 18, 19, 20, 21, 22, 23, 24].

In 25] we present NC-photonic recursion relation by means of Berends and Giele. There we show that, though in NCQED, in contrast to momentum independent color factors of ordinary Yang-Mills theory, the vertex functions depend on momenta, one can derive the recursive relations for currents. In particular, the cosine of a very special combination of momenta comes with the QCD-like currents in recursive relations.

In this paper, we investigate whether similar technique exists for noncommutative QED (NCQED). We use Weyl-van der Waerden spinors [1, 2, 8, 13, 14, and its related calculus with spinorial formalism. The amplitudes of purely photonic and photon-2-fermion processes of noncommutative QED (NCQED) are derived for different helicity configurations of photons. The basic ingredient is the NCQED 
counterpart of Yang-Mills recursion relations by means of Berends and Giele. The explicit solutions of recursion relations for NCQED photonic processes with special helicity configurations are presented.

\section{MHV For Photonic Processes Of NCQED}

The recursion relations for noncommutative photons are presented in [25]. The NCphotonic recursion relation consist two parts. One, as counterpart of color factors of ordinary Yang-Mills theory, consists the cosine of a very special combination of photon's momenta and noncommutativity parameter. The other part is the same of the color-independent part of ordinary Yang-Mills theory, that consists just the momenta. It is shown that since there is no inner product between the cosine part (and the momenta it consists), and as the currents involved in ordinary Yang-MIlls and that of NCQED are similar, we can use all properties of gluonic currents given by [2] in case of NCQED too. The basic tool we use is the so-called spinor formalism. We give expressions for purely photonic and photon-2-fermion processes of NCQED.

First let us introduce the notations; see [25]. We use

$$
\begin{aligned}
& \frac{1}{2} k_{i}^{a} \theta_{a b} k_{j}^{b} \rightarrow i \wedge j \\
& i \wedge k l m \cdots=i \wedge(k+l+m+\cdots)
\end{aligned}
$$

with $\theta_{a b}$ as the noncommutativity parameter. Also we use the symbol "; " as

$$
i j k l \cdots ;=i \wedge(j+k+l+\cdots)+j \wedge(k+l+\cdots)+k \wedge(l+\cdots)+\cdots
$$

and also

$$
\begin{aligned}
\kappa(1, m) \kappa(m+1, k) \kappa(k+1, n) \cdots ; & =\kappa(1, m) \wedge(\kappa(m+1, k)+\kappa(k+1, n)+\cdots) \\
& +\kappa(m+1, k) \wedge(\kappa(k+1 \cdots n)+\cdots)+\cdots(5)
\end{aligned}
$$

with $\kappa^{\mu}(i, j)=\sum_{l=i}^{j} l^{\mu}$. The NCQED recursive relation for purely photonic processes is obtained to be 25 ]

$$
\widehat{J}_{\xi}(1,2, \cdots, n)=(-1)^{n}(i e)^{n-1} \sum_{P(1, \cdots, n)} C\left(123 \cdots n ;+\frac{n-3}{2} \pi\right) J_{\xi}(1, \cdots, n)
$$

in which $e$ as coupling constant, and

$$
\begin{aligned}
C\left(123 \cdots n ;+\frac{n-3}{2} \pi\right) & =\cos (1 \wedge(2+\cdots+n)+2 \wedge(3+\cdots+n)+\cdots \\
& \left.+(n-2) \wedge((n-1)+n)+(n-1) \wedge n+\frac{n-3}{2} \pi\right)
\end{aligned}
$$


The current $J_{\xi}$ is that of [2, 25]

$$
\begin{aligned}
J_{\xi}(1, \cdots, n) & =\frac{1}{\kappa^{2}(1, n)}\left(\sum_{m=1}^{n-1}[J(1, \cdots, m), J(m+1, \cdots, n)]_{\xi}\right. \\
& \left.+\sum_{m=1}^{n-2} \sum_{k=m+1}^{n-1}\{J(1, \cdots, m), J(m+1, \cdots, k), J(k+1, \cdots, n)\}_{\xi}\right)(8
\end{aligned}
$$

in which

$$
\begin{aligned}
{[J(1, \cdots, m), J(m+1, \cdots, n)]_{\xi} } & =2 \kappa(m+1, n) \cdot J(1, \cdots, m) J_{\xi}(m+1, \cdots, n) \\
& -2 \kappa(1, m) \cdot J(m+1, \cdots, n) J_{\xi}(1, \cdots, m) \\
& +(\kappa(1, m)-\kappa(m+1, n))_{\xi} \\
& \times J(1, \cdots, m) \cdot J(m+1, \cdots, n)
\end{aligned}
$$

and

$$
\begin{aligned}
\{J(\alpha), J(\beta), J(\gamma)\}_{\xi} & =J(\alpha) \cdot\left(J(\gamma) J_{\xi}(\beta)-J(\beta) J_{\xi}(\gamma)\right) \\
& -J(\gamma) \cdot\left(J(\beta) J_{\xi}(\alpha)-J(\alpha) J_{\xi}(\beta)\right)
\end{aligned}
$$

in which we used the compact notation $\alpha=(1, \cdots, m), \beta=(m+1, \cdots, k)$ and $\gamma=(k+1, \cdots, n)$. We mention that the current $J_{\xi}$ is the same of [2] appearing in the gluonic recursion relations. This current satisfy the identities

$$
\begin{array}{ll}
\text { (a) } & \kappa(1, n) \cdot J(1, \cdots, n)=0 \\
\text { (b) } & J(1, \cdots, n)=(-)^{n-1} J(n, \cdots, 1) \\
\text { (c) } & \sum_{C y c(1, \cdots, n)} J(1, \cdots, n)=0
\end{array}
$$

The recursion relation include of clock-wise orientation for the labels $1,2, \cdots, n$ that is number of all diagrams occurring in recursion relation; for 3-photon is 3 , and for 4-photon is 10; Fig. 1 and Fig. 2. 


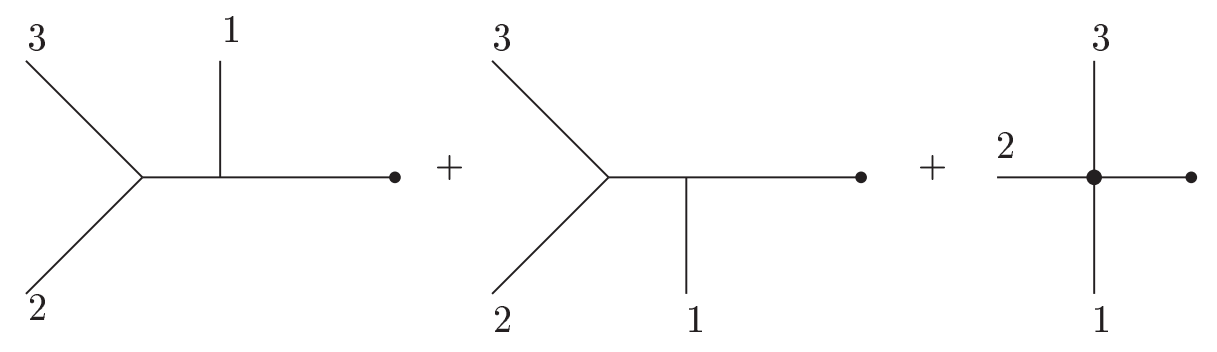

Figure 1: 3-photon case.
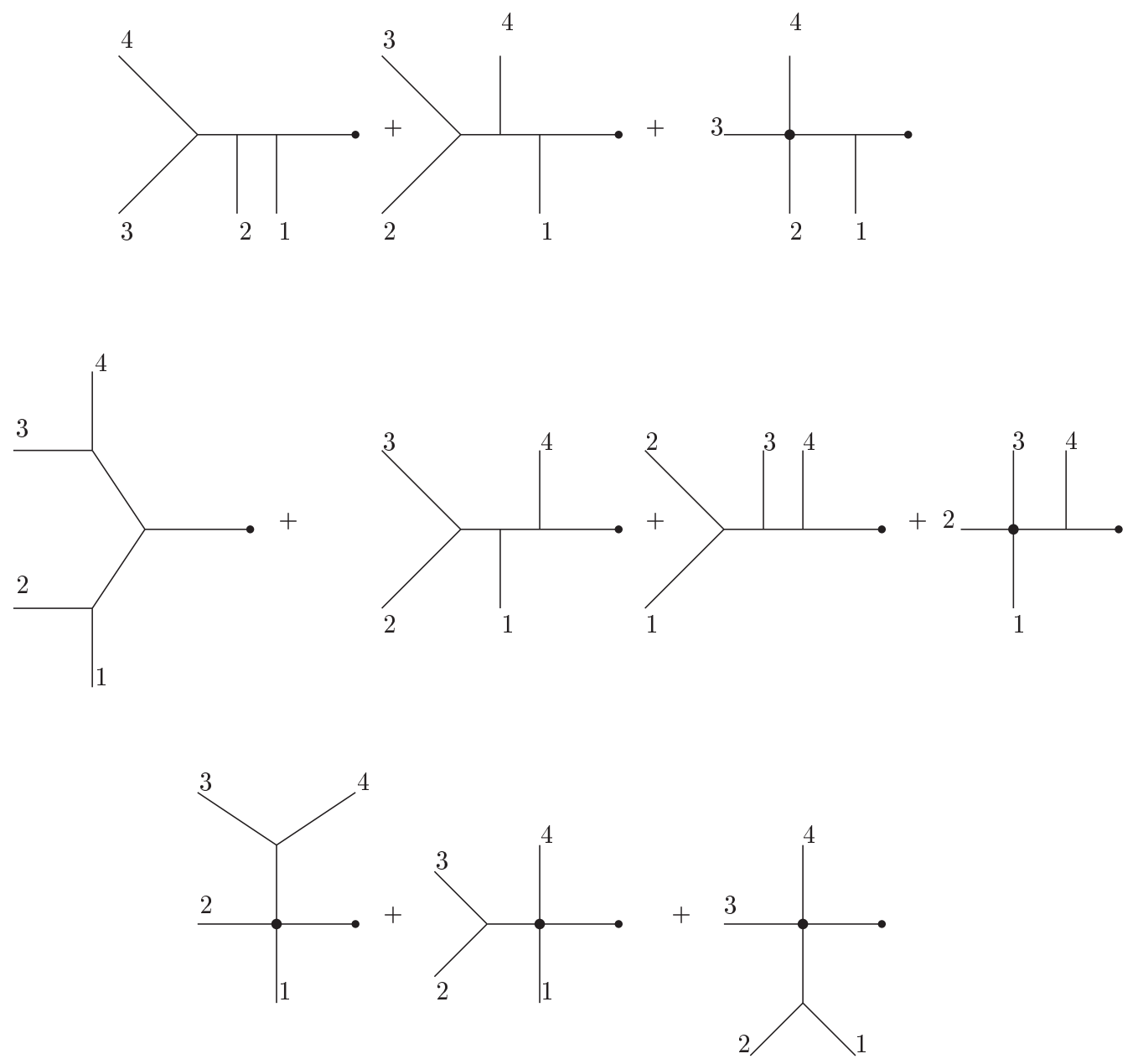

Figure 2: 4-photon case. 


\section{From Currents To Amplitudes And Cross Sections}

Once one is given by the currents, the amplitudes, demanding overall momentum conservation $\kappa^{\mu}(1, n)=0$, are given by

$$
\begin{aligned}
\mathbf{M}(1, \cdots, n)= & \imath \widehat{J}^{\mu}(1, \cdots, n-1) \times\left.\widehat{J}_{\mu}(n) \kappa^{2}(1, n-1)\right|_{\kappa(1, n)=0} \\
= & i(-)^{n-1}(i e)^{n-2} \sum_{P(1, \cdots, n-1)} C\left(1 \cdots n-1 ;+\frac{n-4}{2} \pi\right) \\
& \times\left. J(1, \cdots, n-1) \cdot J(n) \kappa^{2}(1, n-1)\right|_{\kappa(1, n)=0} \\
= & (-1)^{n-1} i^{n} e^{n-2} \sum_{P(1, \cdots, n-1)} C\left(1 \cdots n-1 ;+\frac{n-4}{2} \pi\right) \varepsilon(1, \cdots, n)
\end{aligned}
$$

where $\varepsilon$-function is given by

$$
\varepsilon(1, \cdots, n)=\left.J(1, \cdots, n-1) \cdot J(n) \kappa^{2}(1, n-1)\right|_{\kappa(1, n)=0}
$$

By the properties we know about the currents, we can show the following for $\varepsilon(1, \cdots, n)$ :

1. $\varepsilon$ is invariant under cyclic permutations,

$$
\varepsilon(1, \cdots, n)=\varepsilon(m+1, \cdots, n, 1, \cdots, m)
$$

2. $\varepsilon$ has a reflective property,

$$
\varepsilon(1, \cdots, n)=(-)^{n} \varepsilon(n, \cdots, 1)
$$

3. The sub-cyclic sum equals zero,

$$
\sum_{C y c(1, \cdots, n-1)} \varepsilon(1, \cdots, n)=0
$$

4. The quantity $\varepsilon$ is gauge invariant.

These properties are proven in [2]. For the cross section one must square the amplitude eq.(12), yielding

$$
\begin{aligned}
|\mathbf{M}(1, \cdots, n)|^{2}= & (e)^{2 n-4} \sum_{P(1, \cdots, n-1)} C\left(1 \cdots n-1 ;+\frac{n-4}{2} \pi\right) \varepsilon(1, \cdots, n) \\
& \times \sum_{P(1, \cdots, n-1)} C\left(1 \cdots n-1 ;+\frac{n-4}{2} \pi\right) \varepsilon^{\star}(1, \cdots, n)
\end{aligned}
$$




\section{Solution Of The Recursion Relation For Special Helicity Configurations}

The cross sections often contain contributions from a large number of diagrams. The standard methods of calculation, where an amplitude is squared and summed over all polarizations often become unpractical. For these reasons it became necessary to try other methods of calculation. Most of these procedures calculate amplitudes for specific polarization states of the particles, for which usually helicities are taken. Since at the high energies considered most of the fermion masses can be neglected, the description in terms of helicity states is easily incorporated by using $1 \pm \gamma^{5}$ projections of Dirac spinors. The covariant description of the photon (gluon) helicity in terms of a suitable polarization vector $\epsilon$ is also needed. The recursion relation can be used to calculate step by step any current with a certain number of photons having a specific helicity configuration. For some special helicity configurations the results become so simple that a generalization to an arbitrary number of photons presents itself. The helicity configurations for which this is possible are those in which all photon helicities are the same or all but one are the same. In this way we find $J(1+, 2+, \cdots, n+)$ and $J(1-, 2+, 3+, \cdots, n+)$.

The photonic recursion relation will be solved for two state helicity. The configurations are those where all helicities are the same or all but one are the same. The inner product between 4-vectors has been replaced by spinor contractions, one can choose a gauge for the helicity spinors such that for all polarization vectors $\epsilon_{i}(q) \cdot \epsilon_{j}(q)=0$ ( $q$ is gauge spinor and for more, see Appendix). Through the recursion relation the currents keep this orthogonality property. Thus the 4-vertex contributions vanish, 2, 5. So, the remained purely photonic current is

$$
J_{\mu}(1, \cdots, n)=\frac{1}{\kappa^{2}(1, n)} \sum_{m=1}^{n-1}[J(1, \cdots, m), J(m+1, \cdots, n)]_{\mu}
$$

We can choose following forms polarization state for $J(1+, 2+, \cdots, n+)$

$$
\epsilon_{\mu}^{+}(i, q)=\frac{\left\langle q^{-}\left|\gamma_{\mu}\right| k_{i}^{-}\right\rangle}{\sqrt{2}\left\langle q k_{i}\right\rangle}, \quad 1 \leq i \leq n
$$

Where $q^{\mu}$ is some four-vector different from $k$ and for $J(1-, 2+, \cdots, n+)$

$$
\epsilon_{\mu}^{-}\left(1, k_{2}\right)=-\frac{\left\langle k_{2}^{+}\left|\gamma_{\mu}\right| k_{1}^{+}\right\rangle}{\sqrt{2}\left[k_{2} k_{1}\right]}, \quad \epsilon_{\mu}^{+}\left(i, k_{1}\right)=\frac{\left\langle k_{1}^{-}\left|\gamma_{\mu}\right| k_{i}^{-}\right\rangle}{\sqrt{2}\left\langle k_{1} k_{i}\right\rangle}, \quad 2 \leq i \leq n
$$


where $k_{2}$ in $\epsilon^{-}\left(1, k_{2}\right)$ is as like as $q$ in $\epsilon_{\mu}^{+}(i, q)$, represents the gauge spinor. The recursion relation takes the simple form

$$
\begin{aligned}
J_{\mu}(1, \cdots, n)= & \frac{1}{\kappa^{2}(1, n)} \sum_{m=1}^{n-1}\left(\kappa(m+1, n) \cdot J(1, \cdots, m) J_{\mu}(m+1, \cdots, n)\right. \\
& \left.-\kappa(1, m) \cdot J(m+1, \cdots, n) J_{\mu}(1, \cdots, m)\right)
\end{aligned}
$$

In Appendix we show

$$
J_{\mu}(1+, 2+, \cdots, m+)=\frac{\langle\kappa(1, m) q\rangle\left\langle q^{-}\left|\gamma_{\mu}\right| \kappa^{-}(1, m)\right\rangle}{2^{\frac{m}{2}}\langle\langle q 1, m q\rangle\rangle}
$$

where $\langle\langle q 1, m q\rangle\rangle=\langle q 1\rangle\langle 12\rangle \cdots\langle m-1, m\rangle\langle m q\rangle$. These equations reduce to eq.(19) for one photon. The general case is proven by induction (see Appendix). To evaluate of $J(1-, 2+, \cdots, n+)$, we have

$$
J_{\mu}(1-)=\epsilon_{\mu}^{-}\left(1, k_{2}\right)=-\frac{\left\langle 2^{+}\left|\gamma_{\mu}\right| 1^{+}\right\rangle}{\sqrt{2}[21]}
$$

and we can show that $J_{\mu}(1-, 2+)=0$, So we can show

$$
J_{\mu}(1-2+\cdots n+)=-2^{-\frac{1}{2}} J_{\mu}(2+\cdots n+) \frac{\langle 1 n\rangle[n \kappa(1, n)]\langle 1 \kappa(1, n)\rangle}{\kappa^{2}(1, n-1) \kappa^{2}(1, n)}
$$

This is proven in [2]. Complex conjugation of the currents eq.(22) and eq.(24) gives $J(1-2-\cdots n-)$ and $J(1+2-\cdots n-)$.

Since we have solved the recursion relation for currents in cases of specific helicity configurations we can calculate amplitudes for these situations as well. We do this for n-photon scattering with and without the production of other particles.

From the currents we make $\varepsilon$-functions and from them the helicity amplitudes according to eq.(12) and eq.(13). With the explicit expression for $J^{\mu}(2+\cdots n+)$ in eq.(22) we have

$$
\begin{aligned}
\varepsilon(1 \pm 2+\cdots n+) & =\left.\kappa^{2}(2, n) \epsilon_{\mu}^{ \pm}(1,2) J^{\mu}(2+\cdots n+)\right|_{\kappa(1, n)=0} \\
& =\left.\kappa^{2}(2, n) \epsilon_{\mu}^{ \pm}(1,2) \frac{\langle\kappa(1, m) q\rangle\left\langle q^{-}\left|\gamma_{\mu}\right| \kappa^{-}(1, m)\right\rangle}{2^{\frac{m}{2}}\langle\langle q 1, m q\rangle\rangle}\right|_{\kappa(1, n)=0}=0
\end{aligned}
$$

The vanishing of this $\varepsilon$-function is due to the overall momentum conservation, which leads to a vanishing $\kappa^{2}(2, n)$ (because, $\kappa(1, n)=0 \rightarrow \kappa(2, n)=-k_{1} \rightarrow \kappa^{2}(2, n)=$ $\left.k_{1}^{2}=0\right)$. With the cyclic symmetry of the $\varepsilon$-function, also the $\varepsilon$-function with one 
negative helicity in an arbitrary position vanishes. The helicity amplitude then vanishes as well

$$
\mathbf{M}(1 \pm 2+\cdots n+)=0
$$

The first non-trivial helicity amplitude is $\mathbf{M}(1-2-3+\cdots n+)$, for which we have

$$
\varepsilon(1-2-3+\cdots n+)=\left.\kappa^{2}(2, n) \epsilon_{\mu}^{-}(1,3) J^{\mu}(2-3+\cdots n+)\right|_{\kappa(1, n)=0}
$$

This is not vanishing for conserved momentum, because $J^{\mu}(2-3+\cdots n+)$ has $\frac{1}{\kappa^{2}(2, n)}$ so becomes

$$
\begin{aligned}
\varepsilon(1-2-3+\cdots n+)= & \left(-\frac{\left\langle 3^{+}\left|\gamma_{\mu}\right| 1^{+}\right\rangle}{\sqrt{2}[31]}\right) J^{\mu}(3+\cdots n+) \\
& \left.\left(-2^{-\frac{1}{2}} \frac{\langle 2 n\rangle[n \kappa(2, n)]\langle 2 \kappa(2, n)\rangle}{\kappa^{2}(2, n-1)}\right)\right|_{\kappa(1, n)=0} \\
= & \left(-\frac{\left\langle 3^{+}\left|\gamma_{\mu}\right| 1^{+}\right\rangle}{\sqrt{2}[31]}\right)\left(\frac{\langle\kappa(3, n) 2\rangle\left\langle 2^{-}\left|\gamma_{\mu}\right| \kappa^{-}(3, n)\right\rangle}{2^{\frac{n-3}{2}}\langle\langle 23, n 2\rangle\rangle}\right) \\
& \times\left.\left(-2^{-\frac{1}{2}} \frac{\langle 2 n\rangle[n \kappa(2, n)]\langle 2 \kappa(2, n)\rangle}{\kappa^{2}(2, n-1)}\right)\right|_{\kappa(1, n)=0} \\
= & 2^{(1-n) / 2} \frac{\langle 12\rangle^{4}}{\langle\langle 12, n 1\rangle\rangle}
\end{aligned}
$$

In the general case and from the recursion relation, we find [2]

$$
\begin{aligned}
& \varepsilon(1-3+\cdots m+2-(m+1)+\cdots n+) \\
& =2^{(1-n) / 2} \frac{\langle 12\rangle^{4}}{\langle 13\rangle\langle 34\rangle \cdots\langle m 2\rangle\langle 2(m+1)\rangle \cdots\langle n 1\rangle}
\end{aligned}
$$

which gives the amplitude

$$
\begin{aligned}
& \mathbf{M}(1-, 2-, \cdots, n+) \\
& \quad=(-)^{n-1} i^{n} e^{n-2} 2^{\frac{1-n}{2}}\langle 12\rangle^{4} \sum_{P(1, \cdots, n-1)} C\left(1 \cdots n-1 ;+\frac{n-4}{2} \pi\right) \frac{1}{\langle\langle 12, n 1\rangle\rangle}
\end{aligned}
$$

We mention that the recursion relation for $n$-photon scattering process in $\mathrm{U}(1)$ noncommutative space, is very similar to recursion relation for $n$-gluon scattering case in $\mathrm{SU}(3)$ on ordinary space. By this, we drive photons process scattering amplitudes for special cases in helicity. The main observation is that the helicity configuration is responsible factor for non-vanishing of scattering 


\subsection{MHV Amplitudes For Fermion-Photon Interactions Of NCQED}

We derive recursion relation for electron and positron of NCQED in [25]. We can write electron-photon current in following equation

$$
\widehat{J}(Q, 1, \cdots, n)=e^{n} \sum_{P(1, \cdots, n)} \exp (i \kappa(1, n) Q ;-i \kappa(1, n) ;) J(Q, 1, \cdots, n)
$$

where

$$
J(Q, 1, \cdots, n)=\frac{-1}{\not+k(1, n)-m_{0}} \sum_{m=0}^{n-1} h(n, m) J(Q, 1, \cdots, m) X(m+1, \cdots, n)
$$

in which

$$
h(n, m)=\frac{1}{2}(-1)^{n-m}(i)^{n-m-1}\left(\mathrm{e}^{-i \frac{n-m-3}{2} \pi}+(-1)^{n-m-1} \mathrm{e}^{i \frac{n-m-3}{2} \pi}\right)
$$

with $Q$ and $m_{0}$ as electron's momentum and mass respectively. The fermion case in NCQED is different by quark case in QCD, for reason, we must evaluate helicity amplitudes step by step.

We know electrons obey free Dirac equation and these have ordinary operator

projections, so in following calculations we keep same gauge spinor $(Q$ - electron's momentum) for all photons and we define $\kappa(1, i ; Q)=Q+\kappa(1, i)$ and $Q i=Q+$ $k_{i}$ also $k(1, i ; Q)=\emptyset+\sum_{l=1}^{i} k_{l}$ and $\left|\kappa^{ \pm}(1, i ; Q)\right\rangle\left\langle\kappa^{ \pm}(1, i ; Q)|=| Q^{ \pm}\right\rangle\left\langle Q^{ \pm}|+|\right.$ $\left.\kappa^{ \pm}(1, i)\right\rangle\left\langle\kappa^{ \pm}(1, i)\right|$, in spinorial language

$$
\widehat{J}(Q+)=\left\langle Q^{+}\right|
$$

and

$$
\begin{aligned}
\widehat{J}(Q+, 1+) & =-\frac{1}{\kappa^{2}(1 ; Q)}\left\langle Q^{+}\right| \notin^{+}(1, Q) \kappa(1 ; Q) \\
& =-\frac{1}{\kappa^{2}(1 ; Q)}\left\langle Q^{+}\right|\left(+\frac{\left\langle Q^{-}\left|\gamma^{\mu}\right| 1^{-}\right\rangle}{\sqrt{2}\langle Q 1\rangle} \gamma_{\mu}\right)\left(\sum_{\lambda= \pm}\left|\kappa^{\lambda}(1 ; Q)\right\rangle\left\langle\kappa^{\lambda}(1 ; Q)\right|\right)
\end{aligned}
$$

One mentions

$$
\left\langle Q^{\lambda_{1}}\left|\gamma^{\mu}\right| 1^{\lambda_{2}}\right\rangle \gamma_{\mu}=2\left|1^{\lambda_{2}}\right\rangle\left\langle Q^{\lambda_{1}}|+2| Q^{-\lambda_{1}}\right\rangle\left\langle 1^{\lambda_{2}}\right|
$$

and so

$$
\widehat{J}(Q+, 1+)=-\sqrt{2} \frac{\langle Q \kappa(1 ; Q)\rangle}{\langle 1 Q\rangle\langle Q 1\rangle}\left\langle\kappa(1 ; Q)^{+}\right|
$$


For the next case we have

$$
\begin{aligned}
\widehat{J}(Q+, 1+, 2+) & =-\frac{1}{\kappa^{2}(1,2 ; Q)}\left(h(2,0) J(Q+) J^{+}(1+, 2+)+h(2,1) J(Q+, 1+)\right. \\
& \left.\times \oint^{+}(2, Q)\right) \kappa(1,2 ; Q)=-\frac{1}{(q 2)^{2}}(h(2,0) A+h(2,1) B)
\end{aligned}
$$

where

$$
\begin{aligned}
A & =\left\langle Q^{+}\left|\left(\langle\kappa(1,2) Q\rangle \frac{\left\langle Q^{-}\left|\gamma^{\mu}\right| \kappa^{-}(1,2)\right\rangle}{2\langle Q 1\rangle\langle 12\rangle\langle 2 Q\rangle} \gamma_{\mu}\right) \sum_{\lambda= \pm}\right| \kappa^{\lambda}(1,2 ; Q)\right\rangle\left\langle\kappa^{\lambda}(1,2 ; Q)\right| \\
& =\frac{\langle\kappa(1,2) Q\rangle[Q \kappa(1,2)]\langle Q \kappa(1,2 ; Q)\rangle}{\langle Q 1\rangle\langle 12\rangle\langle 2 Q\rangle}\left\langle\kappa^{+}(1,2 ; Q)\right|
\end{aligned}
$$

and

$$
\begin{aligned}
B & =\left(-\frac{\sqrt{2}\langle Q \kappa(1 ; Q)\rangle}{\langle 1 Q\rangle\langle Q 1\rangle}\left\langle\kappa^{+}(1 ; Q)\right|\right)\left(\frac{\left\langle Q^{-}\left|\gamma^{\mu}\right| 1^{-}\right\rangle}{\sqrt{2}\langle Q 1\rangle} \gamma_{\mu}\right) \sum_{\lambda= \pm}\left|\kappa^{\lambda}(1,2 ; Q)\right\rangle\left\langle\kappa^{\lambda}(1,2 ; Q)\right| \\
& =-2 \frac{\langle Q \kappa(1 ; Q)\rangle[\kappa(1 ; Q) 2]\langle Q \kappa(1,2 ; Q)\rangle}{\langle Q 1\rangle\langle 1 Q\rangle\langle Q 2\rangle}\left\langle\kappa^{+}(1,2 ; Q)\right|
\end{aligned}
$$

Noting

$$
\langle Q \kappa(1 ; Q)\rangle[\kappa(1 ; Q) 2]=\left\langle Q^{-} \kappa^{+}(1 ; Q)\right\rangle\left\langle\kappa^{+}(1 ; Q) 2^{-}\right\rangle=-\left\langle Q^{-}|\not 2| 2^{-}\right\rangle
$$

which from momentum conservation we have $\kappa(1,2 ; Q)=0 \rightarrow \kappa(1 ; Q)=-2$ so

$$
\langle Q \kappa(1 ; Q)\rangle[\kappa(1 ; Q) 2]=-\left\langle Q^{-}|\not 2| 2^{-}\right\rangle=0
$$

giving $\mathrm{B}=0$. So we get

$$
\widehat{J}(Q+, 1+, 2+)=-\frac{1}{\kappa^{2}(1,2 ; Q)} h(2,0) A=\frac{\langle Q \kappa(1,2 ; Q)\rangle}{\langle 1 Q\rangle\langle 12\rangle\langle 2 Q\rangle}\left\langle\kappa^{+}(1,2 ; Q)\right|
$$

The above can be generalized to

$$
\widehat{J}(Q+, 1+, 2+, \cdots, n+)=(-1)^{n} 2^{\frac{2-n}{2}} \frac{\langle Q \kappa(1, n ; Q)\rangle}{\langle\langle 1 Q, n Q\rangle\rangle}\left\langle\kappa^{+}(1, n ; Q)\right|
$$


We prove it by induction. It is correct for $m<n$ so

$$
\begin{aligned}
& \widehat{J}(Q+, 1+, 2+, \cdots, n+) \\
& =-\frac{1}{\kappa^{2}(1, n ; Q)} \sum_{m=0}^{n-1} h(n, m) J(Q+, 1+, \cdots, m+) J^{+}((m+1)+, \cdots, n+) \kappa(1, n ; Q) \\
& =-\frac{1}{\kappa^{2}(1, n ; Q)} \sum_{m=0}^{n-1} h(n, m)(-1)^{m} 2^{\frac{m-2}{2}} \frac{\langle Q \kappa(1, m ; Q)\rangle}{\langle\langle 1 Q, m Q\rangle\rangle}\left\langle\kappa^{+}(1, m ; Q)\right| \\
& \times \frac{\langle\kappa(m+1, n) Q\rangle\left\langle Q^{-}\left|\gamma_{\mu}\right| \kappa^{-}(m+1, n)\right\rangle}{2^{\frac{n-m}{2}}\langle\langle Q(m+1), n Q\rangle\rangle} \gamma^{\mu}\left(\sum_{\lambda= \pm}\left|\kappa^{\lambda}(1, n ; Q)\right\rangle\left\langle\kappa^{\lambda}(1, n ; Q)\right|\right) \\
& =-\frac{1}{\kappa^{2}(1, n ; Q)} \sum_{m=0}^{n-1} h(n, m)(-1)^{m} 2^{\frac{m-2}{2}} \frac{\langle\kappa(m+1, n) Q\rangle\langle Q \kappa(1, m ; Q)\rangle}{\langle\langle 1 Q, m Q\rangle\rangle} \\
& {[\kappa(1, m ; Q) \kappa(m+1, n)]\left\langle Q^{-}\right| \times \frac{2}{2^{\frac{n-m}{2}}\langle\langle Q(m+1), n Q\rangle\rangle}} \\
& \times\left(\sum_{\lambda= \pm}\left|\kappa^{\lambda}(1, n ; Q)\right\rangle\left\langle\kappa^{\lambda}(1, n ; Q)\right|\right)
\end{aligned}
$$

but

$$
\begin{aligned}
& \langle Q \kappa(1, m ; Q)\rangle[\kappa(1, m ; Q) \kappa(m+1, n)]=\left\langle Q^{-} \kappa^{+}(1, m ; Q)\right\rangle \\
& \times\left\langle\kappa^{+}(1, m ; Q) \kappa^{-}(m+1, n)\right\rangle=\left\langle Q^{-}|\kappa(1, m ; Q)| \kappa^{-}(m+1, n)\right\rangle
\end{aligned}
$$

which from momentum conservation we have $\kappa(1, m ; Q)+\kappa(m+1, n)=0 \rightarrow$ $\kappa(1, m ; Q)=-\kappa(m+1, n)$. So all above terms vanish for $m=1$ to $n-1$, but for $m=0$ we have

$$
\begin{aligned}
& \widehat{J}(Q+, 1+, 2+, \cdots, n+) \\
& =-\frac{1}{\kappa^{2}(1, n ; Q)} h(n, 0)\left\langle Q^{+}\right|\left(\sum_{\lambda= \pm}\left|\kappa^{\lambda}(1, n ; Q)\right\rangle\left\langle\kappa^{\lambda}(1, n ; Q)\right|\right) \\
& \times\left(\frac{\langle\kappa(1, n) Q\rangle\left\langle Q^{-}\left|\gamma_{\mu}\right| \kappa^{-}(1, n)\right\rangle}{2^{\frac{n}{2}}\langle\langle Q 1, n Q\rangle\rangle} \gamma^{\mu}\right) \\
& =-\frac{1}{\kappa^{2}(1, n ; Q)} h(n, 0)[Q \kappa(1, n)]\langle Q \kappa(1, n ; Q)\rangle\langle\kappa(1, n) Q\rangle \frac{\left\langle\kappa^{+}(1, n ; Q)\right|}{2^{\frac{n-2}{2}}\langle\langle Q 1, n Q\rangle\rangle} \\
& =-\frac{h(n, 0)\langle Q \kappa(1, n ; Q)\rangle}{2^{\frac{n-2}{2}}\langle\langle Q 1, n Q\rangle\rangle}\left\langle\kappa^{+}(1, n ; Q)\right|
\end{aligned}
$$


in which

$$
\left.-h(n, 0) 2^{\frac{2-n}{2}}=2^{\frac{-n}{2}}(-1)^{n+1} i^{n-1}\left(e^{-i \frac{n-3}{2} \pi}+(-1)^{n-1} e^{i \frac{n-3}{2} \pi}\right)\right)
$$

For any $n$ (odd or even), we have $i^{n-1}\left(e^{-i \frac{n-3}{2} \pi}+(-1)^{n-1} e^{i \frac{n-3}{2} \pi}\right)=-2$ so

$$
-h(n, 0) 2^{\frac{2-n}{2}}=(-1)^{n} 2^{\frac{2-n}{2}}
$$

This completes the proof. For

$$
\begin{aligned}
\widehat{J}(Q+, 1-)= & -\frac{1}{\kappa^{2}(1 ; Q)}\left\langle Q^{+}\right| \notin^{-}(1, Q) \kappa(1 ; Q) \\
= & -\frac{1}{\kappa^{2}(1 ; Q)}\left\langle Q^{+}\right|\left(-\frac{\left\langle Q^{+}\left|\gamma^{\mu}\right| 1^{+}\right\rangle}{\sqrt{2}[Q 1]} \gamma_{\mu}\right)\left(\sum_{\lambda= \pm}\left|\kappa^{\lambda}(1 ; Q)\right\rangle\left\langle\kappa^{\lambda}(1 ; Q)\right|\right) \\
= & -\frac{1}{\kappa^{2}(1 ; Q)}\left\langle Q^{+}\right|\left(-\frac{2\left|1^{+}\right\rangle\left\langle Q^{+}|+2| Q^{-}\right\rangle\left\langle 1^{-}\right|}{\sqrt{2}[Q 1]}\right) \\
& \times\left(\sum_{\lambda= \pm}\left|\kappa^{\lambda}(1 ; Q)\right\rangle\left\langle\kappa^{\lambda}(1 ; Q)\right|\right) \\
= & \sqrt{2} \frac{1}{\kappa^{2}(1 ; Q)}[Q \kappa(1 ; Q)]\langle\kappa(1 ; Q) 1\rangle \frac{1}{[Q 1]} \\
= & \sqrt{2} \frac{1}{\kappa^{2}(1 ; Q)}\left\langle Q^{+}|\kappa(1 ; Q)| 1^{-}\right\rangle \frac{1}{[Q 1]} \\
& =0
\end{aligned}
$$

and

$$
\begin{aligned}
\widehat{J}(Q+, 1-, 2+) & =-\frac{1}{\kappa^{2}(1,2 ; Q)}\left(h(2,0) J(Q+) \not(1-, 2+)+h(2,1) J(Q+, 1-) \oint^{+}(2, Q)\right. \\
& \left.+h(2,1) J(Q+, 2+) \ddagger^{-}(1, Q)\right) \kappa(1,2 ; Q) \\
& =-\frac{1}{\kappa^{2}(1,2 ; Q)}(h(2,0) A+h(2,1)(B+C))
\end{aligned}
$$

But, $B$ and $A$ vanish and from (24) we get to

$$
\widehat{J}(Q+, 1-, 2+)=-\frac{h(2,1) C}{\kappa^{2}(1,2 ; Q)}
$$


where

$$
\begin{aligned}
C & =\left(\frac{\langle Q \kappa(2 ; Q)\rangle}{\langle Q 1\rangle\langle 12\rangle\langle 2 Q\rangle}\left\langle\kappa^{+}(2 ; Q)\right|\right) \\
& \times\left(-\frac{\left\langle Q^{+}\left|\gamma^{\mu}\right| 1^{+}\right\rangle}{\sqrt{2}[Q 1]} \gamma_{\mu}\right)\left(\sum_{\lambda= \pm}\left|\kappa^{\lambda}(1,2 ; Q)\right\rangle\left\langle\kappa^{\lambda}(1,2 ; Q)\right|\right) \\
& =-2 \frac{\langle Q \kappa(2 ; Q)\rangle[\kappa(2 ; Q) Q]\langle 1 \kappa(1,2 ; Q)\rangle}{\sqrt{2}[Q 1]\langle Q 1\rangle\langle 12\rangle\langle 2 Q\rangle}\left\langle\kappa^{+}(1,2 ; Q)\right|
\end{aligned}
$$

One mentions

$$
\begin{aligned}
\langle Q \kappa(2 ; Q)\rangle[\kappa(2 ; Q) Q] & =\left\langle Q^{-} \kappa^{+}(2 ; Q)\right\rangle\left\langle\kappa^{+}(2 ; Q) Q^{-}\right\rangle \\
=\left\langle Q^{-}|k(2 ; Q)| Q^{+}\right\rangle=\left\langle Q^{-}|-1| Q^{+}\right\rangle & =-\langle Q 1\rangle[1 Q]
\end{aligned}
$$

so

$$
\begin{aligned}
\widehat{J}(Q+, 1-, 2+) & =\sqrt{2} \frac{h(2,1)}{\kappa^{2}(1,2 ; Q)} \frac{\langle Q 1\rangle[1 Q]\langle 1 \kappa(1,2 ; Q)\rangle}{[Q 1]\langle Q 1\rangle\langle 12\rangle\langle 2 Q\rangle}\left\langle\kappa^{+}(1,2 ; Q)\right| \\
& =-\sqrt{2} \frac{h(2,1)}{\kappa^{2}(1,2 ; Q)} \frac{\langle Q 1\rangle\langle 1 \kappa(1,2 ; Q)\rangle}{\langle\langle Q 1,2 Q\rangle\rangle}\left\langle\kappa^{+}(1,2 ; Q)\right|
\end{aligned}
$$

in which $h(2,1)=1$. So we have

$$
\widehat{J}(Q+, 1-, 2+)=\frac{-\sqrt{2}}{\kappa^{2}(1,2 ; Q)} \frac{\langle Q 1\rangle\langle 1 \kappa(1,2 ; Q)\rangle}{\langle\langle Q 1,2 Q\rangle\rangle}\left\langle\kappa^{+}(1,2 ; Q)\right|
$$

It is generalized to

$$
\widehat{J}(Q+, 1-, 2+, \cdots, n+)=\frac{(-1)^{n-1} 2^{\frac{n-1}{2}}}{\kappa^{2}(1, n ; Q)} \frac{\langle Q 1\rangle\langle 1 \kappa(1, n ; Q)\rangle}{\langle\langle Q 1, n Q\rangle\rangle}\left\langle\kappa^{+}(1, n ; Q)\right|
$$

We mention that despite the difference between the recursion relations of NCQED and QCD cases, the helicity amplitude of special configuration (all photons and fermion have positive helicity) we approach to same result. Based on this observation, the generalization we suggest seems reasonable, for which we shall present the proof in a forthcoming paper. 


\section{A Spinor Formalism}

The spinor helicity formalism for massless vector bosons [8, 10, 11] is largely responsible for the existence of extremely compact representations of tree and loop partial amplitudes in QCD. It introduces a new set of kinematic objects, spinor products, which neatly capture the collinear behavior of these amplitudes. A (small) price to pay is that automated simplification of large expressions containing these objects is not always straightforward, because they obey nonlinear identities. Here we will review the spinor helicity formalism and some of the key identities. We begin with massless fermions. Positive and negative energy solutions of the massless Dirac equation are identical up to normalization conventions. One way to see this is to note that the positive and negative energy projection operators, $\Lambda_{+}(k) \sim u(k) \cdot \bar{u}(k)$ and $\Lambda_{-}(k) \sim v(k) \cdot \bar{v}(k)$, are both proportional to $k$ in the massless limit. Thus the solutions of definite helicity, $u_{ \pm}(k)=\frac{1}{2}\left(1 \pm \gamma_{5}\right) u(k)$ and $v_{ \pm}(k)=\frac{1}{2}\left(1 \pm \gamma_{5}\right) v(k)$, can be chosen to be equal to each other. (For negative energy solutions, the helicity is the negative of the chirality or $\gamma_{5}$ eigenvalue.) A similar relation holds between the conjugate spinors $\bar{u}_{ \pm}(k)=\frac{1}{2} \bar{u}(k)\left(1 \pm \gamma_{5}\right)$ and $\bar{v}_{ \pm}(k)=\frac{1}{2} \bar{v}(k)\left(1 \pm \gamma_{5}\right)$. Since we will be interested in amplitudes with a large number of momenta, and we use the shorthand notation $\left|i^{ \pm}\right\rangle=\left|k_{i}^{ \pm}\right\rangle=u_{ \pm}\left(k_{i}\right)=v_{\mp}\left(k_{i}\right)$ and $\left\langle i^{ \pm}\right|=\left\langle k_{i}^{ \pm}\right|=\bar{u}_{ \pm}\left(k_{i}\right)=\bar{v}_{\mp}\left(k_{i}\right)$ and we define the basic spinor products by $\langle i j\rangle=\left\langle i^{-} \mid j^{+}\right\rangle=\bar{u}_{-}\left(k_{i}\right) u_{+}\left(k_{j}\right)$ and $[i j]=\left\langle i^{+} \mid j^{-}\right\rangle=\bar{u}_{+}\left(k_{i}\right) u_{-}\left(k_{j}\right)$. The helicity projection implies that products like $\left\langle i^{+} \mid j^{+}\right\rangle$vanish. For numerical evaluation of the spinor products, it is useful to have explicit formulae for them, for some representation of the Dirac $\gamma$ matrices. In the Dirac representation,

$$
\gamma^{0}=\left(\begin{array}{cc}
1 & 0 \\
0 & -1
\end{array}\right) \quad \gamma^{i}=\left(\begin{array}{cc}
0 & \sigma^{i} \\
-\sigma^{i} & 0
\end{array}\right) \quad \gamma^{5}=\left(\begin{array}{cc}
0 & 1 \\
1 & 0
\end{array}\right)
$$

The spinor products are, up to a phase, square roots of Lorentz products. Well see that the collinear limits of massless gauge amplitudes have this kind of squareroot singularity, which explains why spinor products lead to very compact analytic representations of gauge amplitudes, as well as improved numerical stability. We would like the spinor products to have simple properties under crossing symmetry, i.e. as energies become negative. We define $[i j]$ through the identity [14]

$$
\langle i j\rangle[j i]=\left\langle i^{-} j^{+}\right\rangle\left\langle j^{+} i^{-}\right\rangle=\operatorname{tr}\left(\frac{1}{2}\left(1-\gamma_{5}\right) k_{i} k_{j}\right)=2 k_{i} \cdot k_{j}=s_{i j}
$$


We also have the useful identities

- Gordon identity and projection operator:

$$
\left\langle i^{ \pm}\left|\gamma^{\mu}\right| i^{ \pm}\right\rangle=2 k_{i}^{\mu} \quad\left|i^{ \pm}\right\rangle\left\langle i^{ \pm}\right|=\frac{1}{2}\left(1 \pm \gamma_{5}\right) k_{i}
$$

- Antisymmetry:

$$
\langle i j\rangle=-\langle j i\rangle \quad[i j]=-[j i] \quad\langle i j\rangle=[i j]=0
$$

- Fierz rearrangement

$$
\left\langle i^{+}\left|\gamma_{\mu}\right| j^{+}\right\rangle\left\langle k^{+}\left|\gamma^{\mu}\right| l^{+}\right\rangle=2[i k]\langle l j\rangle
$$

- Charge conjugation of current

$$
\left\langle i^{+}\left|\gamma^{\mu}\right| j^{+}\right\rangle=\left\langle j^{-}\left|\gamma^{\mu}\right| i^{-}\right\rangle
$$

- Schouten identity

$$
\langle i j\rangle\langle k l\rangle=\langle i k\rangle\langle j l\rangle+\langle i l\rangle\langle k j\rangle
$$

In an $n$-point amplitude, momentum conservation, $\sum_{i=1}^{n} k_{i}^{\mu}=0$, provides one more identity

$$
\sum_{i=1, i \neq j, k}^{n}[j i]\langle i k\rangle=0
$$

For a massless photon and with momentum $k^{\mu}$ we have in the axial gauge

$$
\sum_{\text {polarization }} \epsilon^{\mu} \epsilon^{\star \nu}=-g^{\mu \nu}+\frac{\left(q^{\mu} k^{\nu}+k^{\mu} q^{\nu}\right)}{q \cdot k}
$$

where $q^{\mu}$ is some four-vector different from $k^{\mu}$. The two degrees of freedom in the spin-1 field will be described with the usual right and left-oriented helicity vectors 14. These helicity vectors $\epsilon_{ \pm}$have the following properties [2, 8, 14]

$$
\begin{array}{r}
\epsilon_{ \pm} \cdot \epsilon_{\mp}^{\star}=0 \quad \begin{array}{r}
k \cdot \epsilon_{ \pm}=0 \quad q \cdot \epsilon_{ \pm}=0 \\
\epsilon_{ \pm}^{\dagger}=\epsilon_{\mp} \quad \epsilon_{ \pm} \cdot \epsilon_{\mp}=-1
\end{array}
\end{array}
$$

By these four properties in the next step, is to introduce a spinor representation for the polarization vector for a massless gauge boson of definite helicity 1 ,

$$
\epsilon_{ \pm}^{\mu}(k, q)= \pm \frac{\left\langle q^{\mp}\left|\gamma^{\mu}\right| k^{\mp}\right\rangle}{\sqrt{2}\left\langle q^{\mp} \mid k^{ \pm}\right\rangle}
$$

This obeys all properties relations and the new spinor $q$ is called the gauge spinor and may be chosen any spinor except for $k$. Making another choice of the gauge spinor is a gauge transformation of the spin-1 field. 


\section{B Photonic Process}

In spinor formalism, 4-vertex vanishes, because the polarization vector has same gauge spinor, so

$$
\epsilon^{+}(i, q) \cdot \epsilon^{+}(j, q) \sim\left\langle q^{-}\left|\gamma_{\mu}\right| i^{-}\right\rangle \cdot\left\langle q^{-}\left|\gamma^{\mu}\right| j^{-}\right\rangle=2[i j]\langle q q\rangle=0
$$

Consider the two equal helicity case ( $q$ is gauge spinor)

$$
\begin{aligned}
J_{\mu}(1+, 2+) & =\frac{1}{\kappa^{2}(1,2)}\left(\frac{1}{2}\left\langle 2^{ \pm}\left|\gamma_{\nu}\right| 2^{ \pm}\right\rangle \frac{\left\langle q^{-}\left|\gamma^{\nu}\right| 1^{-}\right\rangle}{\sqrt{2}\langle q 1\rangle} \frac{\left\langle q^{-}\left|\gamma_{\mu}\right| 2^{-}\right\rangle}{\sqrt{2}\langle q 2\rangle}\right. \\
& \left.-\frac{1}{2}\left\langle 1^{ \pm}\left|\gamma_{\nu}\right| 1^{ \pm}\right\rangle \frac{\left\langle q^{-}\left|\gamma^{\nu}\right| 2^{-}\right\rangle}{\sqrt{2}\langle q 2\rangle} \frac{\left\langle q^{-}\left|\gamma_{\mu}\right| 1^{-}\right\rangle}{\sqrt{2}\langle q 1\rangle}\right)
\end{aligned}
$$

but, $\kappa^{2}(1,2)=2[21]\langle 12\rangle$ so

$$
\begin{aligned}
J_{\mu}(1+, 2+) & =\frac{\left([21]\langle q 2\rangle\left\langle q^{-}\left|\gamma_{\mu}\right| 2^{-}\right\rangle-[12]\langle q 1\rangle\left\langle q^{-}\left|\gamma_{\mu}\right| 1^{-}\right\rangle\right)}{2[21]\langle 12\rangle\langle q 1\rangle\langle q 2\rangle} \\
& =\frac{-\left(\langle q 2\rangle\left\langle q^{-}\left|\gamma_{\mu}\right| 2^{-}\right\rangle+\langle q 1\rangle\left\langle q^{-}\left|\gamma_{\mu}\right| 1^{-}\right\rangle\right)}{2\langle 12\rangle\langle q 1\rangle\langle 2 q\rangle} \\
& =\frac{\left(\langle 2 q\rangle\left\langle q^{-}\left|\gamma_{\mu}\right| 2^{-}\right\rangle+\langle 1 q\rangle\left\langle q^{-}\left|\gamma_{\mu}\right| 1^{-}\right\rangle\right)}{2\langle 12\rangle\langle q 1\rangle\langle 2 q\rangle} \\
& =\frac{\left\langle q^{-}\left|\gamma_{\mu}\right| 2^{-}\right\rangle\langle 2 q\rangle+\left\langle q^{-}\left|\gamma_{\mu}\right| 1^{-}\right\rangle\langle 1 q\rangle}{2\langle 12\rangle\langle q 1\rangle\langle 2 q\rangle} \\
& =\frac{\left\langle q^{-}\left|\gamma_{\mu}\right| 2^{-}\right\rangle\left\langle 2^{-} q^{+}\right\rangle+\left\langle q^{-}\left|\gamma_{\mu}\right| 1^{-}\right\rangle\left\langle 1^{-} q^{+}\right\rangle}{2\langle 12\rangle\langle q 1\rangle\langle 2 q\rangle} \\
& =\frac{\left\langle q^{-}\left|\gamma_{\mu} 2\right| q^{+}\right\rangle+\left\langle q^{-}\left|\gamma_{\mu} \AA\right| q^{+}\right\rangle}{2\langle 12\rangle\langle q 1\rangle\langle 2 q\rangle} \\
& =\frac{\langle\kappa(1,2) q\rangle\left\langle q^{-}\left|\gamma_{\mu}\right| \kappa^{-}(1,2)\right\rangle}{2\langle q 1\rangle\langle 12\rangle\langle 2 q\rangle}
\end{aligned}
$$

This is generalizable to

$$
J_{\mu}(1+, 2+, \cdots, m+)=\frac{\langle\kappa(1, m) q\rangle\left\langle q^{-}\left|\gamma_{\mu}\right| \kappa^{-}(1, m)\right\rangle}{2^{\frac{m}{2}}\langle\langle q 1, m q\rangle\rangle}
$$

where $\langle\langle q 1, m q\rangle\rangle=\langle q 1\rangle\langle 12\rangle \cdots\langle m-1, m\rangle\langle m q\rangle$. The equation reduces to eq.(19)

for one photon. The general equation is proven by induction; suppose eq. (171) to be 
valid for $m<n$ then using eq.(171) we find

$$
\begin{aligned}
& J_{\mu}(1+, 2+, \cdots, n+)=\frac{1}{\kappa^{2}(1, n)} \sum_{m=1}^{n-1}( \\
& \kappa_{\nu}(m+1, n) \frac{\langle\kappa(1, m) q\rangle\left\langle q^{-}\left|\gamma^{\nu}\right| \kappa^{-}(1, m)\right\rangle}{2^{\frac{m}{2}}\langle\langle q 1, m q\rangle\rangle} \frac{\langle\kappa(m+1, n) q\rangle\left\langle q^{-}\left|\gamma_{\mu}\right| \kappa^{-}(m+1, n)\right\rangle}{2^{\frac{n-m}{2}}\langle\langle q(m+1), n q\rangle\rangle} \\
& \left.-\kappa_{\nu}(1, m) \frac{\langle\kappa(m+1, n) q\rangle\left\langle q^{-}\left|\gamma^{\nu}\right| \kappa^{-}(m+1, n)\right\rangle}{2^{\frac{n-m}{2}}\langle\langle q(m+1), n q\rangle\rangle} \frac{\langle\kappa(1, m) q\rangle\left\langle q^{-}\left|\gamma_{\mu}\right| \kappa^{-}(1, m)\right\rangle}{2^{\frac{m}{2}}\langle\langle q 1, m q\rangle\rangle}\right) \\
& =\frac{1}{\kappa^{2}(1, n)} \sum_{m=1}^{n-1} \frac{\mathbb{A}-\mathbb{B}}{2^{\frac{n}{2}}\langle\langle q 1, n q\rangle\rangle}
\end{aligned}
$$

But, from Gordon identity

$$
\begin{aligned}
& \langle\kappa(1, m) q\rangle \kappa_{\nu}(m+1, n)\left\langle q^{-}\left|\gamma^{\nu}\right| \kappa^{-}(1, m)\right\rangle \\
& =\langle\kappa(1, m) q\rangle \frac{1}{2}\left\langle\kappa^{ \pm}(m+1, n)\left|\gamma_{\nu}\right| \kappa^{ \pm}(m+1, n)\right\rangle\left\langle q^{-}\left|\gamma^{\nu}\right| \kappa^{-}(1, m)\right\rangle \\
& =\langle\kappa(1, m) q\rangle[\kappa(m+1, n) \kappa(1, m)]\langle q \kappa(m+1, n)\rangle
\end{aligned}
$$

and by similar way

$$
\begin{aligned}
& \kappa_{\nu}(1, m)\left\langle q^{-}\left|\gamma^{\nu}\right| \kappa^{-}(m+1, n)\right\rangle \\
& =\frac{1}{2}\left\langle\kappa^{ \pm}(1, m)\left|\gamma_{\nu}\right| \kappa^{ \pm}(1, m)\right\rangle\left\langle q^{-}\left|\gamma^{\nu}\right| \kappa^{-}(m+1, n)\right\rangle \\
& =[\kappa(1, m) \kappa(m+1, n)]\langle q \kappa(1, m)\rangle
\end{aligned}
$$

so

$$
\begin{aligned}
\mathbb{A} & =\langle\kappa(1, m) q\rangle[\kappa(m+1, n) \kappa(1, m)]\langle q \kappa(m+1, n)\rangle \\
& \times\langle\kappa(m+1, n) q\rangle\left\langle q^{-}\left|\gamma_{\mu}\right| \kappa^{-}(m+1, n)\right\rangle
\end{aligned}
$$

and

$$
\begin{aligned}
\mathbb{B} & =\langle\kappa(m+1, n) q\rangle[\kappa(1, m) \kappa(m+1, n)]\langle q \kappa(1, m)\rangle \\
& \times\langle\kappa(1, m) q\rangle\left\langle q^{-}\left|\gamma_{\mu}\right| \kappa^{-}(1, m)\right\rangle
\end{aligned}
$$


when

$$
\begin{aligned}
\mathbb{A}-\mathbb{B}= & \langle\kappa(1, m) q\rangle[\kappa(m+1, n) \kappa(1, m)]\langle q \kappa(m+1, n)\rangle \\
\times & \langle\kappa(m+1, n) q\rangle\left\langle q^{-}\left|\gamma_{\mu}\right| \kappa^{-}(m+1, n)\right\rangle \\
- & (-\langle q \kappa(m+1, n)\rangle)(-[\kappa(m+1, n) \kappa(1, m)])(-\langle\kappa(1, m) q\rangle) \\
\times & \langle\kappa(1, m) q\rangle\left\langle q^{-}\left|\gamma_{\mu}\right| \kappa^{-}(1, m)\right\rangle \\
= & \langle\kappa(1, m) q\rangle[\kappa(m+1, n) \kappa(1, m)]\langle q \kappa(m+1, n)\rangle \\
\times & \left(\langle\kappa(m+1, n) q\rangle\left\langle q^{-}\left|\gamma_{\mu}\right| \kappa^{-}(m+1, n)\right\rangle+\right. \\
& \left.\langle\kappa(1, m) q\rangle\left\langle q^{-}\left|\gamma_{\mu}\right| \kappa^{-}(1, m)\right\rangle\right)
\end{aligned}
$$

Similar to eq.(170)

$$
\begin{aligned}
\mathbb{A}-\mathbb{B} & =\langle\kappa(1, m) q\rangle[\kappa(m+1, n) \kappa(1, m)]\langle q \kappa(m+1, n)\rangle \\
& \times\langle\kappa(1, n) q\rangle\left\langle q^{-}\left|\gamma_{\mu}\right| \kappa^{-}(1, n)\right\rangle
\end{aligned}
$$

giving

$$
J_{\mu}(1+, \cdots, n+)=\frac{1}{2^{\frac{n}{2}} \kappa^{2}(1, n)}\langle\kappa(1, n) q\rangle\left\langle q^{-}\left|\gamma_{\mu}\right| \kappa^{-}(1, n)\right\rangle P_{1}^{n-1}
$$

where

$$
P_{1}^{n-1}=\sum_{m=1}^{n-1} \frac{\langle\kappa(1, m) q\rangle[\kappa(m+1, n) \kappa(1, m)]\langle q \kappa(m+1, n)\rangle}{\langle\langle q 1, m q\rangle\rangle\langle\langle q(m+1), n q\rangle\rangle}
$$

for which one can show

$$
P_{1}^{n-1}=\frac{\kappa^{2}(1, n)}{\langle\langle q 1, n q\rangle\rangle}
$$

To evaluate $J(1-, 2+, \cdots, n+)$, we have

$$
J_{\mu}(1-)=\epsilon_{\mu}^{-}\left(1, k_{2}\right)=-\frac{\left\langle 2^{+}\left|\gamma_{\mu}\right| 1^{+}\right\rangle}{\sqrt{2}[21]}
$$

and we can show that $J_{\mu}(1-, 2+)=0$, and

$$
\begin{aligned}
J_{\mu}(1-2+3+) & =\frac{1}{\kappa^{2}(1,3)}\left(-\kappa_{\nu}(2,3) \frac{\left\langle 2^{+}\left|\gamma^{\nu}\right| 1^{+}\right\rangle}{\sqrt{2}[21]} J_{\mu}(2+3+)\right) \\
& =\frac{-1}{\kappa^{2}(1,3)}\left\langle\kappa^{ \pm}(2,3)\left|\gamma_{\nu}\right| \kappa^{ \pm}(2,3)\right\rangle \frac{\left\langle 2^{+}\left|\gamma^{\nu}\right| 1^{+}\right\rangle}{\sqrt{2}[21]} J_{\mu}(2+3+)
\end{aligned}
$$


Noting (71) we get to

$$
\begin{aligned}
J_{\mu}(1-, 2+, 3+) & =\frac{-1}{\kappa^{2}(1,3)} \frac{[\kappa(2,3) 2]\langle 12\rangle\langle 1 \kappa(2,3)\rangle}{\sqrt{2}[21]\langle 12\rangle} J_{\mu}(2+3+) \\
& =\frac{-1}{\sqrt{2}} \frac{\left\langle 1^{-}|(2+\not)| 2^{-}\right\rangle\langle 12\rangle}{\kappa^{2}(1,3) \kappa^{2}(1,2)} J_{\mu}(2+3+) \\
& =\frac{-1}{\sqrt{2}} \frac{\langle 13\rangle[32]\rangle\langle 12\rangle}{\kappa^{2}(1,3) \kappa^{2}(1,2)} J_{\mu}(2+3+)
\end{aligned}
$$

The induction conjecture for $l \geq 3$ is given by

$$
J_{\mu}(1-2+\cdots l+)=-2^{-\frac{1}{2}} J_{\mu}(2+\cdots l+) \sum_{m=3}^{l} \frac{\langle 1 m\rangle[m \kappa(2, m)]\langle 1 \kappa(2, m)\rangle}{\kappa^{2}(1, m-1) \kappa^{2}(1, m)}
$$

this is reduced to

$$
J_{\mu}(1-2+\cdots n+)=-2^{-\frac{1}{2}} J_{\mu}(2+\cdots n+) \frac{\langle 1 n\rangle[n \kappa(1, n)]\langle 1 \kappa(1, n)\rangle}{\kappa^{2}(1, n-1) \kappa^{2}(1, n)}
$$

This is proven in [2]. Complex conjugation of the currents eq.(171) and eq. (866) gives $J(1-2-\cdots n-)$ and $J(1+2-\cdots n-)$.

\section{References}

[1] S. Dittmaier, Phys. Rev. D59 (1999) 016007, hep-ph/9805445.

[2] F. A. Berends and W. T. Giele, Nucl. Phys. B306 (1988) 759; Nucl. Phys. B313 (1989) 595; Nucl. Phys. B294 (1987) 700.

[3] F. Cachazo and P. Svrcek, PoS RTN2005 (2005) 004, hep-th/0504194 G. Georgiou and V. Khoze, JHEP 0405 (2004)070, hep-th/0404072.

[4] K. J. Ozeren and W. J. Stiriling, JHEP 0511 (2005) 016, hep-th/0509063.

[5] R. Kleiss and W. J. Stirling, Phys. Lett. B 179 (1986) 159; and Phys. Lett. B 179 (1986) 2459; R. Kleiss and W. J. Stirling, Nucl. Phys. B 262 (1985) 235;

[6] F. Cachazo, P. Svrcek and E. Witten, JHEP 0409 (2004) 006, hep-th/0403047.

[7] R. Britto, F. Cachazo and B. Feng, Nucl. Phys. B 715 (2005) 499, hep-th/0412308. 
[8] F. A. Berends, R. Kleiss, P. de Causmaecker, R. Gastmans and T. T. Wu, Phys. Lett. B 103 (1981) 124; F. A. Berends, R. Kleiss, P. de Causmaecker, R. Gastmans, W. Troost and T. T. Wu, Nucl. Phys. B 206 (1982) 61; F. A. Berends, P. de Causmaecker, R. Gastmans, R. Kleiss, W. Troost and T. T. Wu, Nucl. Phys. B 239 (1984) 382; Nucl. Phys. B 239 (1984) 395; Nucl. Phys. B 264 (1986) 243; Nucl. Phys. B 264 (1986) 265.

[9] J.-B. Wu and C.-J. Zhu, JHEP 0407 (2004) 032, hep-th/0406085, JHEP 0409 (2004) 063, hep-th/0406146; L. J. Dixon, E. W. N. Glover and V. Khoze, JHEP 0412 (2004) 070, hep-th/0411092.

[10] Z. Xu, D. H. Zhang and L. Chang, Nucl. Phys. B291 391 (1987).

[11] J. F. Gunion and Z. Kunst, Phys. Lett. 161B 333 (1985).

[12] E. Witten, Commun. Math. Phys. 252 (2004) 189-258, hep-th/0312171.

[13] D.A. Kosower, Phys. Rev. D 71 (2005) 045007, hep-th/0406175.

[14] L. J. Dixon, E. W. N. Glover and V. Khoze, JHEP 0412 (2004)015, hep-th/0411092; T. G. Brithwright, E. W. N. Glover, V. Khoze and P. Marquard, JHEP 0505 (2005) 013, hep-ph/0503063.

[15] A. Ilderton, Nucl. Phys. B 742 (2006) 176, hep-th/0512007.

[16] S. Parke and T. Taylor, Phys. Rev. Lett. 56 (1986) 2459.

[17] N. Seiberg and E. Witten, JHEP 9909 (1999) 032, hep-th/9908142 A. Connes, M. R. Douglas, and A. Schwarz, JHEP 9802 (1998) 003, hep-th/9711162; M. R. Douglas and C. Hull, JHEP 9802 (1998) 008, hep-th/9711165.

[18] M. R. Douglas and N. A. Nekrasov, "Noncommutative Field Theory," Rev. Mod. Phys. 73 (2001) 977, hep-th/0106048.

[19] H. Arfaei and M. H. Yavartanoo, "Phenomenological Consequences Of Noncommutative QED," hep-th/0010244 J. L. Hewett, F. J. Petriello, and T. G. Rizzo, Phys. Rev. D64 (2001) 075012, hep-ph/0010354; P. Mathews, Phys. Rev. D63 (2001) 075007, hep-ph/0011332; S.-W. Baek, D. K. Ghosh, X.-G. He, and W. Y. P. Hwang, Phys. Rev. D64 (2001) 056001, hep-ph/0103068, T. M. Aliev, O. Ozcan, and M. Savci, Eur. Phys. J. C27 (2003) 447, hep-ph/0209205 H. Grosse and Y. Liao, Phys. Rev. D64 (2001) 115007, hep-ph/0105090. 
[20] M. Chaichian, M. M. Sheikh-Jabbari, and A. Tureanu, Phys. Rev. Lett. 86 (2001) 2716, hep-th/0010175 Eur. Phys. J. C36 (2004) 251, hep-th/0212259

[21] N. Chair and M. M. Sheikh-Jabbari, Phys. Lett. B504 (2001) 141, hep-th/0009037; M. Haghighat, S. M. Zebarjad, and F. Loran, Phys. Rev. D66 (2002) 016005, hep-ph/0109105; M. Haghighat and F. Loran, Phys. Rev. D67 (2003) 096003, hep-th/0206019 M. Caravati, A. Devoto, and W. W. Repko, Phys. Lett. B556 (2003) 123, hep-ph/0211463.

[22] N. Mahajan, "Noncommutative QED And Gamma Gamma Scattering," hep-ph/0110148.

[23] G. Berrino, S. L. Cacciatori, A. Celi, L. Martucci, and A. Vicini, Phys. Rev. D67 (2003) 065021, hep-th/0210171; C. A. de S. Pires, J. Phys. G30 (2004) B41, hep-ph/0410120, S. M. Lietti and C. A. de S. Pires, Eur. Phys. J. C35 (2004) 137, hep-ph/0402034 T. Mariz, C. A. de S. Pires, and R. F. Ribeiro, Int. J. Mod. Phys. A18 (2003) 5433, hep-ph/0211416 S. I. Kruglov, Annales Fond. Broglie 27 (2002) 343, hep-th/0110059 Electromagn. Phenom. 3 (2003) 18, quant-ph/0204137; Annales Fond. Broglie 26 (2001) 125, math-ph/0110008.

[24] M. Chaichian, P. Presnajder, M.M. Sheikh- Jabbari and A. Tureanu, Eur.Phys.J. C29 (2003) 413-432, hep-th/0107055, and M. M. Sheikh-Jabbari, J. High Energy Phys. 9906 (1999) 015, hep-th/9903107, and I. F. Riad and M. M. Sheikh-Jabbari, J. High Energy Phys. 0008 (2000) 045, hep-th/0008132, and H. Arfaei and M. M. Sheikh-Jabbari, Nucl. Phys. B526 (1998) 278, hep-th/9709054.

[25] A. Jafari, "Recursive Relations For Processes With $n$ Photons Of Noncommutative QED," hep-th/0607141. 